\title{
USO DE ANTIDEPRESIVOS PARA EL TRATAMIENTO DE LOS SÍNTOMAS CLIMATÉRICOS
}

\section{Ulsing antidepressant drugs for treating menopausal symptoms}

Mónica Flores-Ramos, M.D.*, Jesús Rafael Aguilera-Pérez, M.D.**

Recibido: octubre 12/10 - Aceptado: abril 28/11

\section{RESUMEN}

Introducción: los síntomas vasomotores se reportan con frecuencia durante la peri y posmenopausia, y afectan de forma importante la calidad de vida de las mujeres que los sufren. Los estrógenos continúan siendo el tratamiento de elección para estos síntomas, sin embargo, en algunas mujeres este tipo de tratamiento está contraindicado, mientras que otras mujeres simplemente no aceptan usarlo. El objetivo del presente trabajo es hacer una revisión de estudios clínicos que evalúan la eficacia de los antidepresivos en el tratamiento de los síntomas vasomotores.

Materiales y métodos: se realizó una revisión en las bases de datos Medline vía PubMed, EBSCO y Ovid. Se incluyeron artículos, publicados entre 2002 y 2011, de ensayos clínicos aleatorizados, meta-análisis y revisiones sistemáticas que evaluaron la eficacia de fármacos antidepresivos en el tratamiento de síntomas vasomotores.

Resultados: se observa que los antidepresivos ayudan a aliviar los síntomas vasomotores e inciden favorablemente en los síntomas neuropsiquiátricos propios del climaterio, tales como la irritabilidad,

\footnotetext{
* Investigadora en Ciencias Médicas. Instituto Nacional de Perinatología "Isidro Espinosa de los Reyes". México D.F. (México). Correo electrónico: flores_ramos@hotmail.com

** Jefe del Departamento de Peri y posmenopausia. Instituto Nacional de Perinatología “Isidro Espinosa de los Reyes”. México D.F. (México).
}

las alteraciones del sueño y la labilidad emocional. Los estudios en relación a la fisiopatología de los bochornos soportan la hipótesis de que los antidepresivos pueden ser útiles para su tratamiento. Dentro del grupo de medicamentos antidepresivos existe evidencia para el uso de sertralina, fluoxetina, citalopram y venlafaxina.

Conclusión: el uso de antidepresivos puede ser útil en el tratamiento de los síntomas vasomotores relacionados con la menopausia. Debemos considerar aquellos que tengan un perfil farmacológico noradrenérgico y serotoninérgico.

Palabras clave: antidepresivos, climaterio, bochornos, síntomas vasomotores, tratamiento.

\section{SUMMARY}

Introduction: vasomotor symptoms are frequently reported during the peri and postmenopausal periods and drastically affect the quality of life of those women who suffer them. Estrogens continue being the treatment of choice for such symptoms; however, this type of treatment is contraindicated in some women, whilst other women simply refuse to use them. The present work was aimed at reviewing clinical studies which have evaluated antidepressant drugs' effectiveness regarding the treatment of vasomotor symptoms.

Materials and methods: Medline / PubMed, EBSCO, and Ovid databases were reviewed. 
Articles published between 2002 and 2011 were included, as were randomized clinical assays, metaanalyses and systematic reviews which evaluated antidepressant drugs' effectiveness in treating vasomotor symptoms.

Results: it was observed that antidepressants helped to alleviate vasomotor symptoms and had a favorable effect on neuropsychiatric symptoms related to the menopause, such as irritability, alterations in sleeping patterns and emotional ability. Studies regarding hot flushes' physiopathology supported the hypothesis that antidepressants could be useful for treating them. Evidence regarding the group of antidepressant medicines suggested using sertraline, fluoxetine, citalopram and venlafaxine.

Conclusion: antidepressant drugs could be useful in treating menopause-related vasomotor symptoms; those having a noradrenergic and serotoninergic pharmacological profile should be considered.

Key words: antidepressant drug, menopause, hot flushes, vasomotor symptoms, treatment.

\section{INTRODUCCIÓN}

La sintomatología observada durante el período de la perimenopausia y la posmenopausia es variada, e incluye síntomas vasomotores, alteraciones psicológicas y genitourinarias. En un estudio de cohorte con una muestra amplia (1462 mujeres) se reportó que el 64\% de las mujeres de 54 años de edad, refiere síntomas vasomotores (SVM), característica distintiva de la perimenopausia y posmenopausia. ${ }^{1}$ Dichos síntomas ejercen un efecto negativo sobre la calidad de vida y la capacidad funcional de las mujeres. ${ }^{2}$ En México, en un estudio llevado a cabo por Horna y colaboradores ${ }^{3}$ se evaluó la presencia de diferentes síntomas en una población entre 45 y 65 años de edad, observándose que en las mujeres perimenopáusicas los síntomas más frecuentes eran sudoraciones, calor excesivo, bochornos, dolor de cabeza, resequedad vaginal y dispareunia; mientras que en las mujeres posmenopáusicas se observó con mayor frecuencia una pérdida del interés sexual. Datos internacionales ${ }^{4}$ provenientes de una amplia revisión sistemática, también apoyan que los bochornos y la sudoración son los síntomas más prevalentes durante este período y que se presentan en mujeres de toda clase social; aunque su reporte puede estar influido por características sociodemográficas, roles sociales y actitudes frente a la edad y el final de la vida reproductiva. Independientemente de las diferencias étnicas, los síntomas vasomotores tienen un impacto negativo en la vida de la mujer por lo que es importante reconocerlos y evaluar la necesidad de tratarlos de acuerdo a la individualidad de cada paciente. A pesar de que el tratamiento con estrógenos sigue siendo el más recomendable para las pacientes peri y posmenopáusicas sintomáticas, ${ }^{5}$ no todas las pacientes aceptan tratamiento con hormonas o incluso en algunas está contraindicado su uso. Además, los efectos colaterales que se han observado con el uso de estrogénicos, como el riesgo de cáncer de mama, ${ }^{6}$ han limitado su uso. Por lo anterior, continuamente se estudian diferentes tipos de fármacos para el alivio de los síntomas climatéricos, ${ }^{7}$ en los que se incluyen antidepresivos, antagonistas dopaminérgicos, veraliprida, clonidina, extractos de isoflavona y extractos de soya, entre otros. En el caso de los antidepresivos podemos suponer que además del efecto que puedan tener en los síntomas vasomotores, existe una mejoría en los síntomas neuropsiquiátricos que también ocurren durante el climaterio, como las alteraciones del sueño, la irritabilidad y la tristeza; lo que podría ser de gran utilidad para un tratamiento integral de las pacientes climatéricas.

Aunque no se conoce del todo la fisiopatología de los bochornos, se sabe que en la termorregulación se encuentran implicados neurotransmisores como la serotonina y la noradrenalina, ${ }^{8}$ por lo que el uso de antidepresivos favorecería el alivio de estos síntomas. Sin embargo, aún es poca la evidencia referente a la seguridad en el uso de este tipo de fármacos durante este período en la vida de la mujer. Antoine y colaboradores 9 realizaron una revisión con el objetivo de evaluar 
la seguridad de los tratamientos utilizados para el alivio de los síntomas climatéricos, en pacientes sobrevivientes a cáncer de mama. Los autores analizaron sistemáticamente estudios realizados con tibolona, inhibidores selectivos de la recaptura de serotonina, clonidina, veraliprida, gabapentina, cimicifuga y fitoestrógenos; llegando a la conclusión de que no existen datos disponibles que indiquen la ausencia de efecto dañino de estos medicamentos. Esta revisión incluyó a una población específica, quizá en la que más se ha evaluado el uso de tratamientos diferentes a la terapia hormonal de reemplazo, pero sería también conveniente evaluar qué ocurre en la población general de mujeres peri y posmenopáusicas; desafortunadamente en la actualidad contamos con pocos datos al respecto.

Por lo tanto, la presente revisión tiene como objetivo conocer la eficacia de los fármacos antidepresivos para atenuar los síntomas vasomotores del climaterio, tanto en pacientes con y sin antecedentes de cáncer de mama.

\section{MATERIALES Y MÉTODOS}

Se realizó una revisión de artículos en los que el objetivo era evaluar la eficacia de fármacos antidepresivos en el tratamiento de los síntomas climatéricos. La búsqueda se realizó en las bases de datos de Medline vía PubMed, EBSCO y Ovid. Se incluyeron artículos publicados entre enero de 2002 y enero de 2011, en idioma inglés, de ensayos clínicos aleatorizados, meta-análisis y revisiones sistemáticas. Se utilizaron como palabras de búsqueda: "antidepressants, serotonergic agents, serotonin-norepinephrine reuptake inhibitors, moclobemide, y mirtazapine" en combinación con los términos "vasomotor symptoms", "climacteric symptoms" y "hot flashes". De los artículos encontrados incluimos tanto los que reportan resultados positivos como negativos de la eficacia de éstos fármacos; y dividimos la información de inhibidores selectivos de la recaptura de serotonina, inhibidores de la recaptura de serotonina y noradrenalina y otros antidepresivos para la presentación de los datos.

\section{RESULTADOS}

Incluimos resultados de 23 estudios que evaluaron la eficacia de antidepresivos para el control de síntomas vasomotores. Se excluyeron los estudios que reportaron cambios en éstos síntomas como un resultado secundario y aquéllos que no utilizaron una medición validada para evaluar el cambio en cuanto a número y frecuencia de bochornos. De los estudios incluidos, 16 fueron ensayos clínicos controlados con placebo y los 7 restantes fueron estudios abiertos (en los que no existe grupo control) o simple ciego (en donde el paciente o el observador desconoce qué tratamiento está recibiendo). Los resultados son presentados de acuerdo al perfil farmacológico de los antidepresivos estudiados. Encontramos 12 estudios que utilizaron inhibidores selectivos de la recaptura de serotonina, 7 que emplearon antidepresivos duales y 4 que emplearon otro tipo de antidepresivos.

\section{Inhibidores selectivos de la recaptura de serotonina}

Dentro de esta familia de fármacos existen estudios que evalúan la eficacia para disminuir los síntomas climatéricos. En tales estudios investigan sobre los siguientes medicamentos: paroxetina, sertralina, fluoxetina, citalopram y escitalopram. Loprinzi y colaboradores (2002) realizaron un estudio clínico aleatorizado, doble ciego, de 4 semanas de duración que evaluó la eficacia de fluoxetina para disminuir los bochornos y reportó una utilidad modesta de este fármaco en el tratamiento de los bochornos en mujeres que habían sufrido cáncer de mama. ${ }^{10}$ En este trabajo, el análisis inicial no reportó diferencias estadísticamente significativas; sin embargo, después de cruzar los grupos comparativos y realizar un análisis posterior, la fluoxetina demostró ser mejor que el placebo (24\% más de reducción, $\mathrm{p}=0,02)$. 
En relación a la paroxetina, existe un estudio llevado a cabo por Stearns y colaboradores (2003) en el que se evalúa el tratamiento con paroxetina de liberación controlada (CR), durante 6 semanas a 165 mujeres posmenopáusicas, que habían padecido cáncer de mama y que sufrían al menos dos bochornos diarios. Las 165 pacientes fueron asignadas aleatoriamente a tres grupos de tratamiento: $12,5 \mathrm{mg}$ de paroxetina $(\mathrm{n}=51), 25 \mathrm{mg}$ de paroxetina $(n=58)$, y placebo $(n=56)$. De estas pacientes 160 fueron incluidas en el análisis de eficacia. Los resultados encontrados después de 6 semanas de tratamiento fueron una disminución en el índice compuesto de bochornos del 62,2\% en el grupo de $12,5 \mathrm{mg}$ de paroxetina, $64,6 \%$ en pacientes tratados con $25 \mathrm{mg}$ de paroxetina, en comparación a un 37,8\% de reducción en el grupo de placebo. Esta reducción permaneció significativa después de ajustar el análisis por edad, historia previa de enfermedad o uso de antiestrógenos. ${ }^{11}$ Los mismos autores ${ }^{12}$ habían observado previamente que la preparación habitual de paroxetina de $20 \mathrm{mg}$ producía una reducción en los bochornos de mujeres sobrevivientes a cáncer de mama; sin embargo, ambos estudios se realizaron en una población específica (pacientes tratadas por el antecedente de cáncer de mama), por lo que no es posible generalizar a todo tipo de mujeres climatéricas.

Existen también estudios que se han llevado a cabo en pacientes con menopausia natural y sin terapia hormonal de reemplazo, como el que desarrollaron Gordon y colaboradores (2006), en el cual se evaluaron a 102 mujeres de 40 a 65 años de edad. Se trata de un estudio doble ciego, cruzado, de dos brazos, aleatorizado y controlado con placebo, que evalúa la eficacia de la sertralina en el tratamiento de los bochornos. En los resultados de este estudio se muestra que las pacientes que se encontraban en el brazo de tratamiento con sertralina presentaron una disminución cinco veces mayor en la frecuencia de bochornos, en relación a las pacientes que recibían placebo. No se observó una disminución en la intensidad de los bochornos con este fármaco, sin embargo, el índice de bochornos (frecuencia x severidad) disminuyó notablemente. ${ }^{13}$ Por su parte, Grady y colaboradores (2007) encuentran resultados contrarios en un estudio aleatorizado, doble ciego y controlado con placebo, en el cual evalúan a 99 mujeres entre 40 y 60 años de edad, con 14 o más bochornos al día. Los autores encuentran que después de 6 semanas de tratamiento la disminución en la frecuencia de bochornos resulta igual para el grupo de sertralina (39\%) que para el grupo de placebo (38\%); así mismo, el índice de bochornos es igual para ambos grupos de estudio. Además, los autores encuentran que la sertralina se relaciona con mayor frecuencia a efectos colaterales como malestares gastrointestinales, sequedad de boca y sudoración. ${ }^{14}$ En este mismo sentido, Wu y colaboradores (2009) no demostraron la eficacia de la sertralina para disminuir los bochornos en un estudio clínico aleatorizado, doble ciego, con un grupo control tomando placebo; en el que solamente encontraron mejoría en cuanto al bienestar emocional. ${ }^{15}$

El citalopram, es otro inhibidor selectivo de la recaptura de serotonina (ISRS) que ha sido estudiado en cuanto a su eficacia para el tratamiento de los síntomas climatéricos. Un estudio llevado a cabo por Suvanto-Luukonene y colaboradores (2005) con diseño aleatorizado y doble ciego, de 9 meses de duración, que compara citalopram con fluoxetina y placebo, ${ }^{16}$ no encuentra resultados estadísticamente significativos en cuanto a la diferencias de los ISRS con el placebo en la disminución de los bochornos; incluso sugiere que no debe utilizarse este tipo de fármacos con este propósito. Sin embargo otro estudio reciente ${ }^{17}$ contradice estos resultados. Se trata de un estudio llevado a cabo por Kalay y colaboradores (2007) diseñado con cuatro grupos de tratamiento: 1) citalopram, 2) placebo, 3) citalopram más terapia hormonal; y 4) placebo más terapia hormonal. En este ensayo clínico se incluyeron a 100 mujeres posmenopáusicas que se asignaron al azar a los grupos mencionados. En el caso de mujeres que no pudieran recibir terapia 
hormonal, la asignación fue a los grupos 1 ó 2. Mientras que las mujeres con respuesta inadecuada a la terapia hormonal fueron asignadas a los grupos 3 ó 4. Los autores observaron una disminución de los bochornos, en las quejas psicosociales y en el índice de Kupperman modificado, en todos los grupos. El índice de bochornos mostró una disminución de 37\% en el grupo 1, 13\% en el grupo 2, 50\% en el grupo 3 y 14\% en el grupo 4; lo que denota una disminución mayor en los grupos 1 y 3 en comparación a los grupos 2 y 4, siendo esta diferencia estadísticamente significativa. Los autores concluyen que el citalopram es un tratamiento alternativo eficaz para el alivio de los síntomas vasomotores.

Otro estudio con citalopram es el realizado por Barton y colaboradores (2010) en el que se evalúa el tratamiento de los síntomas vasomotores en un estudio clínico controlado, aleatorizado, con dosis de 10, 20 y $30 \mathrm{mg}$ de citalopram al día. Este trabajo incluyó a 254 mujeres y evaluó la frecuencia y severidad de los bochornos como medida de eficacia, así como la escala de interferencia diaria de los bochornos. En los resultados se observó la efectividad del citalopram en el tratamiento de los bochornos y buena tolerancia al fármaco, con mejores efectos a dosis de $20 \mathrm{mg}$ diarios. ${ }^{18}$

Soares y colaboradores (2006) evaluaron la eficacia del escitalopram en comparación con la terapia hormonal sustitutiva (estrógenos más progestágenos) para el tratamiento de los síntomas en mujeres perimenopáusicas y posmenopáusicas, por medio de un estudio clínico aleatorizado. Los autores incluyeron a 40 mujeres entre 40 y 60 años de edad, con trastorno depresivo y síntomas relacionados con la menopausia, a quienes trataron con citalopram a dosis de 10 a $20 \mathrm{mg}$ o progesterona más estrógenos ( $5 \mu \mathrm{g}$ de etinilestradiol más $1 \mathrm{mg}$ de acetato de noretindrona al día) durante 8 semanas. Los instrumentos de medición utilizados por los autores fueron la escala de Montgomery-Asberg para depresión y la escala de Greene para síntomas climatéricos. Los autores observaron que el 75\% de las pacientes tratadas con citalopram presentaron remisión de los síntomas depresivos, mientras que solamente el 25\% de las pacientes tratadas con estrógenos más progestágenos mostraron mejoría en este rubro $(p=0,01)$. Para los síntomas relacionados a la menopausia se observó una remisión del 56\% de las mujeres tratadas con escitalopram y un 31,2\% de las mujeres tratadas con terapia hormonal. ${ }^{19}$ Estos resultados sugieren que el escitalopram puede ser una opción de tratamiento para las mujeres con síntomas menopáusicos que tienen alguna contraindicación para el uso de terapias hormonales. Es importante recalcar que este estudio incluye a mujeres deprimidas, lo que pudiera influir en los resultados.

Freeman y colaboradores (2011) realizaron un ensayo clínico aleatorizado, multicéntrico de 8 semanas de duración para evaluar la eficacia y tolerancia del escitalopram en el tratamiento de los bochornos; la dosis utilizada de escitalopram fue de 10 a 20 mg al día y los instrumentos de medición fueron la frecuencia y severidad de los bochornos de acuerdo a diarios de evaluación. Se observó una reducción, en al menos 50\%, de la frecuencia de bochornos del 55\% de las pacientes que tomaron escitalopram y del 35\% de las pacientes incluidas en el grupo con placebo. La disminución en la severidad de los bochornos también fue mayor en las pacientes con escitalopram que en las pacientes con placebo. ${ }^{20}$

Existen pocos estudios en relación a la fluvoxamina para el tratamiento de los síntomas vasomotores. Oishi y colaboradores (2007) realizaron un estudio piloto, abierto, en 22 mujeres a quienes se les administró $50 \mathrm{mg}$ diarios de fluvoxamina para el control de sus síntomas vasomotores. Los instrumentos de medición de eficacia fueron el índice simple de menopausia (SMI) y el cuestionario autoaplicado para depresión (SRQ-D). Los autores no encontraron disminución en las escalas, pero sí una disminución en los síntomas vasomotores después de 6 semanas de tratamiento. Este último estudio presenta importantes limitaciones metodológicas pero introduce un nuevo fármaco 
como alternativa para el tratamiento de los síntomas vasomotores; se requieren de estudios controlados para verificar la eficacia de la fluvoxamina en el tratamiento de síntomas vasomotores. ${ }^{21}$

\section{Inhibidores de la recaptura de serotonina y noradrenalina.}

Dentro de este grupo de fármacos, la venlafaxina es la que más ha sido estudiada, aunque también existen ya reportes del tratamiento de síntomas climatéricos con duloxetina y milnacipram. Ladd y colaboradores (2005), realizaron un estudio abierto en el que se incluyeron a 16 mujeres en fase de climaterio y con diagnóstico de depresión mayor según el manual diagnóstico y estadístico de los trastornos mentales (DSM-IV) que fueron tratadas con venlafaxina de liberación prolongada, durante 8 semanas. Las pacientes fueron evaluadas con la escala de Hamilton para la evaluación de la depresión (HAM-D), la escala de impresión clínica global (CGI) y la escala climatérica de Green (GCS). En este trabajo se encontró que la venlafaxina disminuyó los síntomas depresivos de forma significativa a las dos semanas de tratamiento; el 75\% de las pacientes presentó remisión de la depresión después de las 8 semanas de tratamiento y la puntuación en la GCS disminuyó en un 60\%. Los autores sugieren que la venlafaxina es útil tanto para el tratamiento de los síntomas depresivos como para los síntomas vasomotores del climaterio. ${ }^{22}$

En un estudio abierto de 5 semanas de duración llevado a cabo por Loprinzi y colaboradores (1998) se evaluó a mujeres sobrevivientes a cáncer de mama y hombres con tratamiento de deprivación androgénica, que sufrían bochornos (mínimo 14 por semana). En éste ensayo se observó una disminución de al menos $50 \%$ en el número de bochornos con el tratamiento de 12,5 mg de venlafaxina, dos veces al día en 25 pacientes que completaron las cinco semanas de tratamiento. ${ }^{23}$ Así mismo, se observó una disminución en la incidencia de bochornos severos y muy severos $(\mathrm{p}<0,0002)$ al final del estudio. Los dos estudios descritos previamente fueron abiertos, por lo que los resultados deben ser tomados con reserva; sin embargo, un reporte de otro estudio aleatorizado, controlado con placebo, confirma la eficacia de venlafaxina para el tratamiento de los síntomas vasomotores del climaterio. ${ }^{24}$ Este estudio fue realizado por Loprinzi y colaboradores (2000) en mujeres sobrevivientes a cáncer de mama ó mujeres preocupadas por sufrirlo, renuentes a tratamiento hormonal. De 221 participantes, se obtuvieron los datos de 191 pacientes que fueron asignadas aleatoriamente a cuatro grupos de tratamiento: 1) $37,5 \mathrm{mg}$ diarios de venlafaxina de liberación prolongada, durante 28 días; 2) 37,5 mg diarios de venlafaxina de liberación prolongada, durante 7 días, seguidos de $75 \mathrm{mg}$ de venlafaxina en la misma presentación durante 21 días; 3) 37,5 mg diarios de venlafaxina de liberación prolongada, durante 7 días, 75 mg durante 7 días y 150 mg durante 14 días; y 4) placebo. Los resultados obtenidos de este trabajo demostraron una disminución mayor en el número de bochornos en los grupos de tratamiento que en el grupo placebo (con una diferencia estadísticamente significativa, $\mathrm{p}<0,0001)$, una mejoría significativa de los síntomas depresivos en los grupos de tratamiento con venlafaxina, y un aumento en las puntuaciones de calidad de vida en los grupos de tratamiento en comparación con el grupo placebo. Todas las dosis de venlafaxina fueron efectivas, aunque la presencia de efectos colaterales fue mayor con dosis de $150 \mathrm{mg}$.

La comparación entre venlafaxina y gabapentina realizada por Bordeleau y colaboradores (2010) arroja resultados interesantes. Los autores realizaron un estudio abierto, cruzado, en el que evalúan la preferencia de las pacientes por venlafaxina o gabapentina. Se encuentra que la disminución en las puntuaciones de los índices de bochornos es similar con los dos fármacos (66\%); sin embargo, la mayoría de las pacientes prefirió venlafaxina (68\%), en comparación a gabapentina (32\%). La venlafaxina se asoció con incremento en náusea, pérdida de apetito, constipación, y menores cambios en el 
ánimo; mientras que la gabapentina se relacionó con mareo y aumento del apetito. ${ }^{25}$ Así mismo, el tratamiento con venlafaxina ha sido comparado con la acupuntura en un estudio que realizaron Walker y colaboradores (2010). En este trabajo los autores asignaron de forma aleatoria a 50 pacientes a dos grupos de tratamiento: acupuntura $(n=25)$ o venlafaxina $(n=25)$ durante 12 semanas. Se observó una mejoría significativa con los dos tipos de tratamiento en cuanto a bochornos, síntomas depresivos y otros indicadores de calidad de vida. La incidencia de efectos colaterales fue mayor con venlafaxina que con acupuntura, además después de suspender el tratamiento las pacientes con venlafaxina volvieron a experimentar los bochornos mientras que en el caso de las pacientes tratadas con acupuntura no se observó este efecto. ${ }^{26}$

La desvenlafaxina, un análogo de la venlafaxina, también ha sido estudiada como un agente para el tratamiento de los síntomas vasomotores. Archer y colaboradores (2009) evaluaron su eficacia para disminuir los bochornos en un estudio aleatorizado, doble ciego, con tres grupos de tratamiento: 1) placebo; 2) $100 \mathrm{mg}$ de desvenlafaxina; y 3) $150 \mathrm{mg}$ de desvenlafaxina, durante 26 semanas. Las medidas de eficacia fueron el número y la severidad de los bochornos, así como el número de despertares durante la noche debidos a los bochornos. Se encontró que el número de pacientes que respondieron con una disminución $\geq 50 \%$ y $\geq 75 \%$ en el promedio diario de bochornos fue significativamente superior en los grupos que recibieron desvenlafaxina (100 ó $150 \mathrm{mg} /$ día) que en el grupo de pacientes que recibió placebo. De igual manera, existió una diferencia significativa en cuanto al número de despertares debidos al bochorno entre las pacientes tratadas con desvenlafaxina y el grupo placebo, existiendo en las primeras una disminución mayor de estos. ${ }^{27}$

Otro medicamento con perfil farmacológico dual (inhibidor selectivo de la recaptura de serotonina y noradrenalina) que también ha sido evaluado en cuanto a su eficacia para el tratamiento de los síntomas vasomotores del climaterio es la duloxetina. Joffe y colaboradores (2007) llevaron a cabo un estudio en mujeres posmenopáusicas entre 40 y 60 años de edad, deprimidas y con síntomas vasomotores. Un estudio de 2 semanas de duración, simple ciego, controlado con placebo, seguido de 8 semanas de tratamiento abierto con dosis flexibles de duloxetina (60 a $120 \mathrm{mg}$ al día). De las 30 mujeres que se eligieron para participar, 20 iniciaron la fase de tratamiento con duloxetina y 14 de ellas completaron el estudio. Se encontró una disminución estadísticamente significativa en las puntuaciones de la escala de depresión (Montgomeri- Asberg Depression Rating Scale) después de 8 semanas de tratamiento $(\mathrm{p}<0,001)$. También se observó una mejoría estadísticamente significativa en los síntomas vasomotores, ansiedad, calidad del sueño y dolor. ${ }^{28}$ Los autores sugieren que la duloxetina puede utilizarse en mujeres posmenopáusicas deprimidas; sin embargo, debemos considerar que se trata de un estudio abierto con una muestra pequeña.

\section{Otros antidepresivos}

Existen pocos datos en relación al uso de otros antidepresivos para el tratamiento de los síntomas climatéricos. Un trabajo llevado a cabo por Tarim y colaboradores (2002), evaluó la eficacia de la moclobemida (un inhibidor de la monoaminooxidasa reversible) para disminuir la frecuencia y severidad de los bochornos. Se trató de un ensayo clínico aleatorizado en el que se incluyeron 30 mujeres tratadas con $150 \mathrm{mg} /$ día de moclobemida (grupo 1, $\mathrm{n}=10$ ), $300 \mathrm{mg} /$ día de moclobemida (grupo 2, $\mathrm{n}=11)$ o placebo $(\mathrm{n}=9)$ durante 5 semanas. Los autores encontraron una reducción promedio en el índice de bochornos de 24,4\% en el grupo placebo, en comparación a 69,8\% en el grupo 1 y 35,0\% en el grupo 2. Los investigadores concluyen que la moclobemida es una buena opción de tratamiento; sin embargo, el estudio tiene un grupo limitado de pacientes. ${ }^{29}$ También existe un estudio piloto ${ }^{30}$ realizado por Pérez y colaboradores (2004), en el que evalúan la eficacia y tolerabilidad de la mirtazapina 
en el tratamiento de los síntomas vasomotores. Se trata de un estudio abierto, prospectivo, de cinco semanas de duración, en el que se trataron a 22 mujeres con dosis crecientes de mirtazapina (de 7,5 mg a $30 \mathrm{mg}$ diarios, por la noche). Se reportan datos de 16 pacientes que completaron el estudio y que presentaron una disminución diaria de 52,5\% en el índice de bochornos, y una reducción semanal de 59,5\% en la misma medición. Además, las mujeres reportaron mejoría en los síntomas de tensión, alteraciones del sueño, sudoración anormal y preocupación por los bochornos, así como mejor calidad de vida. A pesar de que se trata de un estudio piloto, los datos muestran una eficacia prometedora del fármaco para aliviar los síntomas climatéricos.

Existe un trabajo que evalúa la eficacia del bupropión (un inhibidor selectivo de la recaptura de serotonina y noradrenalina). Se trata de un análisis realizado por Pérez y colaboradores (2006) en el que reportan resultados desalentadores en el uso de este fármaco para tal fin. Es un estudio piloto, abierto, en el que se incluyeron 21 pacientes tratados inicialmente con 150 mg al día de bupropión y después de 3 días con $300 \mathrm{mg}$ del mismo fármaco, hasta completar 4 semanas. Cinco pacientes abandonaron el estudio a causa de los efectos colaterales. En el resto de pacientes no se observó una reducción importante de los síntomas y no existieron diferencias significativas en relación al placebo. Aunque el tamaño de muestra fue pequeño, los resultados fueron consistentes con el mecanismo de acción del antidepresivo, por lo cual los autores no recomiendan el uso de este fármaco para el tratamiento de los síntomas vasomotores del climaterio. ${ }^{31}$

Por último, el uso de la hierba de San Juan (hypericum perforatum), un antidepresivo proveniente de una raíz, el cual también ha sido estudiado en cuanto a su eficacia para el tratamiento de los síntomas vasomotores, tanto solo como en combinación con cimicifuga racemosa. Uebelhack y colaboradores (2006) realizaron un estudio clínico doble ciego, controlado con placebo de 6 semanas de duración en el que incluyeron 301 mujeres que presentaban síntomas climatéricos, en el que evaluaron la combinación de cimicifuga racemosa con hypericum perforatum para aliviar dichos síntomas. Los autores reportaron una disminución del 50\% en las puntuaciones de la escala de menopausia (Menopause rating scale) en el grupo de tratamiento, en comparación con 19,6\% en el grupo placebo; así mismo, observaron una disminución en la escala de depresión (HAM-D) del 41,8\% en el grupo de tratamiento, frente $12,7 \%$ en el grupo placebo. El tratamiento fue significativamente superior que el placebo en ambas mediciones $(\mathrm{p}<0,001)$, sin diferencias significativas en cuanto a efectos colaterales, valores de laboratorio o tolerancia. ${ }^{32}$

\section{DISCUSIÓN}

A pesar de que la terapia hormonal sustitutiva sigue siendo la primera opción de tratamiento para mujeres sintomáticas en peri y posmenopausia, ahora se cuenta con otras estrategias de tratamiento que han demostrado eficacia en la disminución de síntomas vasomotores. Tal es el caso de los antidepresivos que además disminuyen los síntomas depresivos y ansiosos propios de esta etapa y mejoran la calidad del sueño. La Sociedad Norteamericana de Menopausia y el Colegio Americano de Ginecología y Obstetricia han recomendado que en mujeres con bochornos, entre moderados a severos, relacionados con la menopausia, que manifiesten contraindicaciones para el uso de compuestos que contengan estrógenos, debe considerarse el uso de progestágenos, venlafaxina, paroxetina, fluoxetina o gabapentina. ${ }^{33}$ Sin embargo, es necesario continuar trabajando en esta área para poder proporcionar a nuestras pacientes diferentes opciones cuando su queja principal son los síntomas vasomotores. Los estudios con los que contamos en la actualidad tienen una gran diversidad metodológica, muchos de ellos con limitaciones como son: tamaño pequeño de la muestra, inclusión de mujeres con antecedente de cáncer mamario, inclusión de pacientes del sexo masculino, períodos de evaluación cortos e 
instrumentos de medición diferentes para evaluar efectividad. Por lo anterior, sería conveniente realizar más estudios que puedan evaluar la eficacia de los antidepresivos en el tratamiento de los síntomas vasomotores a largo plazo y con muestras amplias, que incluyan mujeres con menopausia natural o quirúrgica.

Es importante también considerar que algunos de los fármacos antidepresivos son inhibidores potentes del sistema del citocromo P450, como la paroxetina y la fluoxetina, especialmente en mujeres que toman tamoxifen. ${ }^{34}$ Se requieren, además, estudios que evalúen la diferencia entre el uso de inhibidores selectivos de recaptura de serotonina contra el uso de inhibidores duales como la venlafaxina, duloxetina y milnacipram. Por último, es importante considerar los efectos colaterales sexuales de los antidepresivos, ya que se ha observado una alta tasa de disfunción sexual en pacientes en este período de su vida. ${ }^{35} \mathrm{La}$ búsqueda de un fármaco que ayude al tratamiento integral de los síntomas climatéricos es difícil, sin embargo, es importante conocer todas las opciones con las que contamos para poder prescribir adecuadamente a cada paciente, de acuerdo con sus necesidades.

\section{CONCLUSIÓN}

El uso de antidepresivos puede ser útil en el tratamiento de los síntomas vasomotores relacionados con la menopausia. Debemos considerar aquellos que tengan un perfil farmacológico noradrenérgico y serotoninérgico.

\section{REFERENCIAS}

1. Rödström K, Bengtsson C, Lissner L, et al. A longitudinal study of the treatment of hot flushes: the population study of women in Gothenburg during a quarter of a century. Menopause 2002;9:156-61.

2. Blümel JE, Chedraui P, Baron G, Belzares E, Bencosme A, Calle A, et al. A large multinational study of vasomotor symptom prevalence, duration and impact on quality of life in middle-aged women. Menopause 2011 Mar 14. En prensa.
3. Horna A, Romero G, Horna M, Malacara JM, Pérez EL. El perfil sintomático en mujeres peri y posmenopáusicas. Ginec Obstet Mex 2006;74:312-6.

4. Freeman EW, Sherif K. Prevalence of hot flushes and night sweats around the world: a systematic review. Climacteric 2007;10:197-214.

5. Nelson HD. Postmenopausal estrogen for treatment of hot flashes. Clinical applications. JAMA 2004;291:1621-5.

6. Beral V; Million Women Study Collaborators. Breast cancer and hormone-replacement therapy in the Million women study. Lancet 2003;362:419-27.

7. Nelson HD, Vesco KK, Haney E, Fu R, Nedrow A, Miller J, et al. Nonhormonal therapies for menopausal hot flashes, systematic review and meta-analysis. JAMA 2006;295:2057-71.

8. Joffe H, Soares CN, Cohen LS. Assessment and treatment of hot flushes and menopausal mood disturbance. Psychiatr Clin North Am 2003;26:563-80.

9. Antoine C, Liebens F, Carly B, Pastijn A, Rozenberg S. Safety of alternative treatments for menopausal symptoms after breast cancer: a qualitative systematic review. Climacteric 2007;10:23-6.

10. Loprinzi CL, Sloan JA, Perez EA, Quella SK, Stella PJ, Mailliard JA, et al. Phase III evaluation of fluoxetine for treatment of hot flashes. J Clin Oncol 2002;20:1578-83.

11. Stearns V, Beebe K, Iyengar M, Dube E. Paroxetine controlled release in the treatment of menopausal hot flashes: a randomized controlled trial. JAMA 2003;289:2827-34.

12. Stearns V, Isaacs C, Rowland J, Crawford J, Ellis MJ, Kramer R, et al. A pilot trial assessing the efficacy of paroxetine hydrochloride (Paxil) in controlling hot flashes in breast cancer survivors. Ann Oncol 2000;11:17-22.

13. Gordon PR, Kerwin JP, Boesen KG, Senf J. Sertraline to treat hot flashes: a randomized, controlled, double-blind, crossover trial in a general population. Menopause 2006;13:546-8.

14. Grady D, Cohen B, Tice J, Kristof M, Olyaie A, Sawaya GF. Ineffectiveness of sertraline for treatment of menopausal hot flushes: a randomized controlled trial. Obstet Gynecol 2007;109:823-30.

15. Wu MF, Hilsenbeck SG, Tham YL, Kramer R, Elledge RM, Chang JC, et al. The efficacy of sertraline for controlling hot flashes in women with or at high risk 
of developing breast cancer. Breast Cancer Res Treat 2009;118:369-75.

16. Suvanto-Luukkonen E, Koivunen R, Sundströrm H, Bloigu R, Karjalainen E, Häiva-Mällinen L, Tapanainen JS. Citalopram and fluoxetine in the treatment of postmenopausal symptoms: a prospective, randomized, 9-month, placebo controlled, doubleblind study. Menopause 2005;12:18-26.

17. Kalay AE, Demir B, Haberal A, Kalay M, Kandemir O. Efficacy of citalopram on climacteric symptoms. Menopause 2007;14:223-9.

18. Barton DL, LaVasseur BI, Sloan JA, Stawis AN, Flynn KA, Dyar M, et al. Phase III, placebo-controlled trial of three doses of citalopram for the treatment of hot flashes: NCCTG trial N05C9. J Clin Oncol 2010;28:3278-83.

19. Soares CN, Arsenio H, Joffe H, Bankier B, Cassano P, Petrillo LF, et al. Escitalopram versus ethinyl estradiol and norethindrone acetate for symptomatic peri and postmenopausal women: impact on depression, vasomotor symptoms, sleep and quality of life. Menopause 2006;13:780-6.

20. Freeman EW, Guthrie KA, Caan B, Sternfeld B, Cohen L, Joffe H, et al. Efficacy of escitalopram for hot flashes in healthy menopausal women: a randomized controlled trial. JAMA 2011;305:267-74.

21. Oishi A, Mochizuki Y, Otsu R, Inaba N. Pilot study of fluvoxamine treatment for climacteric symptoms in Japanese women. Biopsychosoc Med 2007;1:12.

22. Ladd CO, Newport DJ, Ragan KA, Loughhead A, Stowe $\mathrm{ZN}$. Venlafaxine in the treatment of depressive and vasomotor symptoms in women with perimenopausal depression. Depress Anxiety 2005;22:94-7.

23. Loprinzi CL, Pisanski TM, Fonseca R, Sloan JA, Zahasky KM, Quella SK, et al. Pilot evaluation of venlafaxine hydrochloride for the therapy of hot flashes in cancer survivors. J Clin Oncol 1998;16:2377-81.

24. Loprinzi CL, Kugler JW, Sloan JA, Mailliard JA, LaVasseur BI, Barton DL, et al. Venlafaxine in management of hot flashes in survivors of breast cancer: a randomized controlled trial. Lancet 2000;356:2059-63.

25. Bordeleau L, Pritchard KI, Loprinzi CL, Ennis M, Jugovic O, Warr D, et al. Multicenter, randomized, cross-over clinical trial of venlafaxine versus gabapentin for the management of hot flashes in breast cancer survivors. J Clin Oncol 2010;28:5147-52.
26. Walker EM, Rodríguez AI, Kohn B, Ball RM, Pegg J, Pocock JR, et al. Acupunture versus venlafaxine for the management of vasomotor symptoms in patients with hormone receptor-positive breast cancer: a randomized controlled trial. J Clin Oncol 2010;28:634-40.

27. Archer DF, Dupont CM, Constantine GD, Pickar JH, Olivier S; Study 319 Investigators. Desvenlafaxine for the treatment of vasomotor symptoms associated with menopause: a double-blind, randomized, placebocontrolled trial of efficacy and safety. Am J Obstet Gynecol 2009;200:238.e1-238.e10.

28. Joffe H, Soares CN, Petrillo LF, Viguera AC, Somley B, Koch J, et al. Treatment of depression and menopauserelated symptoms with the serotonin-norepinephrine reuptake inhibitor duloxetine. J Clin Psychiatry 2007;68:943-50.

29. Tarim E, Bagis T, Kilicdag E, Erkanli S, Aslan E, Kuscu E. Moclobemide in the treatment of hot flashes in postmenopausal women. Adv Ther 2002;19:258-65.

30. Perez DG, Loprinzi CL, Barton DL, Pockaj BA, Sloan J, Novotny PJ, et al. Pilot evaluation of mirtazapine for the treatment of hot flashes. J Support Oncol 2004;2:50-6.

31. Pérez DG, Loprinzi CL, Sloan J, Novotny P, Barton D, Carpenter L, et al. Pilot evaluation of bupropion for the treatment of hot flashes. J Palliat Med 2006;9:631-7.

32. Uebelhack R, Blohmer JU, Graubaum HJ, Busch R, Gruenwald J, Wernecke KD. Black cohosh and St John's Wort for Climacteric complaints: a randomized trial. Obstet Gynecol 2006;107:247-55.

33. Stearns V. Serotonergic agents as an alternative to hormonal therapy for the treatment of menopausal vasomotor symptoms. Treat Endocrinol 2006;5:83-7.

34. Caraci F, Crupi R, Drago F, Spina E. Metabolic drug interactions between antidepressants and anticancer drugs: Focus on selective serotonin reuptake inhibitors and hypericum extract. Curr Drug Metab 2011 Mar 14. En prensa.

35. Schnatz PF, Whitehurst SK, O’Sullivan DM. Sexual dysfunction, depression, and axiety among patients of an inner-city menopause clinic. J Womens Health (Larchmt) 2010;19:1843-9. 